\title{
Human hAtg2A protein expressed in yeast is recruited to preautophagosomal structure but does not complement autophagy defects of $\operatorname{atg} 2 \Delta$ strain
}

\author{
Daria Romanyuk, Anna Polak, Agnieszka Maleszewska, Marzena Sieńko, Marcin Grynberg \\ and Teresa Żołądek
}

Institute of Biochemistry and Biophysics, Polish Academy of Sciences, Warszawa, Poland

\begin{abstract}
Yeast Atg2, an autophagy-related protein, is highly conserved in other fungi and has two homologues in humans, one of which is hAtg2A encoded by the hATG2A/ KIAA0404 gene. Region of homology between Atg2 and hAtg2A proteins comprises the C-terminal domain. We used yeast atg2 2 strain to express the GFP-KIAA0404 gene, its fragment or fusions with yeast $A T G 2$, and study their effects on autophagy. The GFP-hAtg2A protein localized to punctate structures, some of which colocalized with Ape1-RFP-marked preautophagosomal structure (PAS), but it did not restore autophagy in atg2 $\Delta$ cells. $\mathrm{N}$-terminal fragment of Atg2 and $\mathrm{N}$-terminal fragment of hAtg2A were sufficient for PAS recruitment but were not sufficient to function in autophagy. Neither a fusion of the N-terminal fragment of hAtg2 A with C-terminal domain of Atg2 nor a reciprocal fusion were functional in autophagy. hAtg2A, in contrast to yeast Atg2, did not show interaction with the yeast autophagy protein Atg9 but both Atg2 proteins showed interaction with Atg18, a phospholipid-binding protein, in two-hybrid system. Moreover, deletion of ATG18 abrogated PAS recruitment of hAtg2A. Our results show that human hAtg2A can not function in autophagy in yeast, however, it is recruited to the PAS, possibly due to the interaction with Atg18.
\end{abstract}

Keywords: autophagy, human hAtg2A protein, yeast Atg2, nitrogen starvation

Received: 05 March, 2011; revised: 02 June, 2011; accepted: 02 August, 2011; available on-line: 29 August, 2011

\section{INTRODUCTION}

Cell growth and death are determined by the presence or absence of specific proteins. Depending on the extracellular conditions, specific balance is maintained between protein synthesis and degradation. Proteins are degraded by two major pathways: the ubiquitin-proteasome pathway (Hershko \& Ciechanover, 1998; Goldberg, 2003) and autophagy (Klionsky \& Ohsumi, 1999; Xie \& Klionsky, 2007). During authophagy cytoplasmic components are enwrapped into an autophagosome and delivered to the vacuole for degradation. In yeast, at least 35 autophagy-related genes ( $A T G$ ) have been found to be essential for autophagy (Klionsky et al., 2003; Kabeya et al., 2007). Many Atg proteins, including Atg2, localize to the pre-autophagosomal structure or phagophore assembly site (PAS; Xie \& Klionsky, 2007), the center of autophagosome formation (Suzuki et al., 2001; Suzuki \& Ohsumi, 2010). In the yeast Saccharomyces cerevisiae autophagy overlaps the cytoplasm-to-vacuole targeting (Cvt) pathway which is responsible for the biosynthetic delivery of aminopeptidase I (Ape1) from the cytoplasm to the vacuole (Klionsky et al., 1992; Lynch-Day \& Klionsky, 2010). The Cvt pathway operates in growth conditions and autophagy is induced by starvation. Many Atg proteins are common to both the Cvt and autophagy pathways.

Atg2 is a peripheral membrane protein of 1592 amino acids (aa) and a predicted molecular mass of $178 \mathrm{kDa}$ (Shintani et al., 2001; Wang et al., 2001) which is localized in the perivacuolar PAS and is also found in the cytosol. Efficient localization of Atg2 to the PAS is dependent on a membrane protein, Atg9, which binds Atg2 (Wang et al., 2001; Suzuki et al., 2001; 2007). Atg2 forms a complex with Atg18 (Obara et al., 2008b) and together they are required for Atg9 recycling between the PAS and non-PAS membraneous compartments (Reggiori et al., 2004; Suzuki et al., 2007). Moreover, Atg2 and Atg18 localize to the PAS interdependently in autophagy-inducing conditions (Suzuki et al., 2007). The domain structure of Atg2 is unknown. A point mutation causing the G83E substitution results in a severe defect in Atg2 function (Shintani et al., 2001).

In higher eukaryotic cells autophagy is mechanistically identical to the process that occurs in yeast (Reggiori \& Klionsky, 2002). In mammalian cells autophagy has multiple physiological functions: it is involved in cell death, tumor suppression, aging, age-associated diseases and neurodegeneration (reviewed in: Bergamini et al., 2004; Gozuacik \& Kimchi, 2004; van der Vaart et al., 2008). Numerous genes for components of the human autophagic machinery have been cloned thanks to their homology to yeast $A T G$ genes and shown to complement autophagy defects of respective yeast mutants (Reggiori \& Klionsky, 2002). The homology of yeast Atg2 and the human autophagy-related protein 2 homolog A encoded by the $h A T G 2 A / K I A A 0404$ gene and further referred to as hAtg2A was pointed out previously (Shintani et al., 2001). The $h A T G 2 A / K L A A 0404$ gene further referred to as KIAA0404 was upregulated in the etoposide-induced apoptosis in HeLa cells and by doxorubicin, an inhibitor of topoisomerase II (Kusama et al., 2009). However, hAtg2A has not been characterized until very

e-mail: teresa@ibb.waw.p

Abbreviations: aa, amino acid; Cvt, cytoplasm-to-vacuole targeting; GFP, green fluorescent protein; orf, open reading frame; PAS, preautophagosomal structure; RFP, red fluorescent protein; S.D., standard deviation 
recently when several hAtg2A-interacting proteins were identified (Behrends et al., 2010).

Yeast is widely used as a model organism to study the function of human proteins and better to understand many human diseases (reviewed in: Foury, 1997; Petranovic \& Nielsen, 2008). Here we studied in silico the similarity of yeast Atg2 protein to proteins from other organisms and investigated whether the human hAtg2A protein expressed in yeast could function in autophagy. We also asked if the C-terminus of Atg2 is required for Atg2 function and/or could be replaced by the C-terminus of hAtg2A, and, conversely, if hAtg2A with the corresponding Atg2 part is functional in autophagy. Moreover, we studied the interaction of Atg 2 and hAtg2A with Atg9 and Atg18 by using the two-hybrid system.

\section{MATERIALS AND METHODS}

Strains, media and growth conditions. The S. cerevisiae strains used were TN125 MAT $\alpha$ ade2 ura3 leu2 his3 trp1 lys2 PHO8::pho8 460, YTS24 atg2::LEU2 PHO8::pho8 $\triangle 60$ (isogenic to TN125) (Shintani et al., 2001) and PJ69-4A MAT a trp1-901 leu2-3, 122 his3-200 ura3-52 gal4 $\Delta$ gal80A LYS2::GAL1-HIS3, GAL2-ADE2 met2::GAL7-lacZ (James et al., 1996). Yeast growth and transformation followed standard procedures (Sherman, 2002). YPD (1\% yeast extract, $1 \%$ peptone, $2 \%$ glucose), SC-leu or SC-leu-his (containing $0.68 \%$ yeast nitrogen base without amino acids, and $20 \mathrm{mg} / 1$ of adenine, uracil and required amino acids minus leucine and histidine) with $2 \%$ glucose as a carbon source were used. For nitrogen starvation cells were shifted to YB-N $(0.17 \%$ yeast nitrogen base without ammonium sulphate and amino acids) containing glucose as a carbon source. For two-hybrid interaction transformants were grown on SC-leu-trp-his with addition of 5-10 mM 3-aminotriazole at $28^{\circ} \mathrm{C}$ for $3-7$ days.

In strains TN125 and YTS24 the LEU2 gene was deleted to anable use of plasmids with the LEU2 marker in further studies and were substituted with a $\operatorname{KanMX}$ cassette by genomic integration of BamHI-digested pLEU2::kanMX (Voth et al., 2003) to produce strains with a leu2::kanMX marker, YPJ2 and YPJ1, respectively.

The ability of cells to form colonies was used to measure their survival under nitrogen starvation conditions. Cells were grown to $\mathrm{OD}_{600}$ about 1 in YPD or $\mathrm{SC}-\mathrm{leu}$, washed once and then resuspended in YB-N to an $\mathrm{OD}_{600}$ about 0.3. At the indicated times, $\mathrm{OD}_{600}$ was measured, aliquots were removed, serially diluted and plated on YPD plates in triplicate. Results shown are representative of two independent experiments.

Plasmids and plasmid construction. The plasmids used in this study were: BS-KIAA0404 (Kazusa DNA Research Institute, Japan), pUG36 (Niedenthal et al., 1996), p415 $5_{\mathrm{GPD} 1}$ [LEU2 marker (Mumberg et al., 1995)], pAPE1-RFP (based on pRS313, kind gift of M. Thumm, University of Gottingen, Germany), YEp-ATG2 (Shintani et al., 2001), pGBDUAtg9 (Reggiori et al., 2005), PS-150 bearing the AD-ATG18 fusion (kind gift of D. J. Klionsky, University of Michigan, USA (Nair et al., 2010)), pGEM (pGEM-TEasy, Promega), pGBT-9 (Clontech) and pACT2 (Clontech).

To express human KIAA0404 gene in yeast, plasmid p415-GFP-KIAA0404 bearing GFP fused with $K I A A 0404$ gene under the constitutive GPD1 promoter was constructed. First the XbaI fragment of pUG36 bearing the GFP gene was transferred to $X b a \mathrm{I}$ sites of p415 ${ }_{\mathrm{GPD} 1}$ plasmid to obtain p415-GFP. Then BamHIEcoRV 6221 bp fragment of a BS-KIAA0404 derivative, in which a BamHI restriction site had been introduced between the MluI and Nsi sites by PCR sitedirected mutagenesis of pGEM-MluI-NsiI, was ligated with BamHI and SmaI-digested p415-GFP. The primers used for in vitro site specific mutagenesis and other PCR reactions are shown in Table S1. To obtain plasmid p415-GFP-KIAA0404-N expressing fragment of hAtg2A protein containing amino acids $1-1561$, plasmid p415-GFP-KIAA0404 was digested with Eco47III and religated.

Plasmid p415-GFP-ATG2 bearing GFP fused to the yeast ATG2 gene was also constructed for control. To this end SphI-SacII DNA fragment containing ATG2 was transferred from YEp-ATG2 to pGEM and pGEM-ATG2 was used for two rounds of in vitro PCR mutagenesis to insert XmaI and SalI restriction sites flanking ATG2 open reading frame (orf). Resulting pGEM-ATG2-XS was sequenced and was digested with XmaI, ends were filled in with Klenow polymerase, and after SalI digestion the fragment bearing ATG2 was isolated and ligated with SmaI and SalIdigested p415-GFP. To obtain p415-GFP-ATG2-N encoding Atg2 protein devoid of C-terminal domain (aa 1-1184), BlpI-StuI fragment of p415-GFP-ATG2 was replaced with similar fragment in which SalI site had been introduced by in vitro PCR mutagenesis and the 1235-bp SalI fragment was removed by SalI digestion and religation.

To analyze the possible function of $\mathrm{hAtg} 2 \mathrm{~A} \mathrm{~N}$ terminal and C-terminal fragments, plasmids expressing appropriate gene fusions, p415-GFP-ATG2-NKIAA0404-C and p415-GFP-KIAA0404-N-ATG2-C, were constructed by fusion-PCR. Fragment B/pI-StuI of p415-GFP-ATG2 was transferred to pGEM to obtain pGAF. pGAF was used as a template for PCR to amplify ATG2 fragment and to add 18 bp overhang of KIAA0404 sequence. This fragment together with PCR-amplified fragment of KIAA0404 encoding C-terminal part of the hAtg2A protein, with $18 \mathrm{bp}$ overhang of ATG2 sequence (Table S1), was used as a template for fusion PCR. Product of fusion PCR was digested with $B / p \mathrm{I}$ and SalI and was used to replace BlpI-SalI fragment of p415-GFP-ATG2 and obtain p415-GFPATG2-N-KIAA0404-C which encodes a fusion of N-terminal part of Atg2 (aa 1-1184) with C-terminal part of hAtg2A (aa 1540-1938). Plasmid p415-GFPKIAA0404-N-ATG2-C encoding fusion protein of N-terminal part of hAtg2A (aa 1-1539) fused with Cterminal part of Atg2 (aa 1185-1592) was obtained by replacing SacII-SalI fragment of p415-GFP- KIAA0404 with SacII and SalI-digested product of fusion PCR. In this fusion-PCR two PCR products were used as templates: one DNA product of 1277 bp amplified by using p415-GFP-ATG2 as a template and ATG2-specific oligonucleotides, one with 18-nucleotide KIAA0404 overhang added, and the other DNA product of 1238 bp amplified by using p415-GFP-KIAA0404 as a template and KIAA0404-specific oligonucleotides, one with 18-nucleotide ATG2 overhang added.

To analyze the physical interaction of proteins by two-hybrid system, plasmids expressing fusions of GALA domains with full lenght ATG2, ATG9 or KIAA0404 were constructed. To obtain pGBT9ATG2, pGEM-ATG2-XS (see above) was digested with XmaI and SalI, and 4794 bp fragment was transferred to pGBT9. To obtain pGBT9-KIAA0404, 7.2- 
Table S1. Oligonucleotides used

\begin{tabular}{|c|c|c|c|}
\hline Name & Sequence & $\begin{array}{l}\text { Nucleotide position } \\
\text { in ATG2, ATG9 or } \\
\text { KIAA0404 orf }\end{array}$ & Description \\
\hline Xma11 & TITITITGATTTCCCGGGAATGGCATTTGG & \multirow{2}{*}{ ATG2 1-12 } & \multirow{2}{*}{ introduce $\mathrm{Xmal}$ site } \\
\hline Xma11rev & CCAAAATGCCATTCCCGGGAAATCAAAAAAAA & & \\
\hline Sal11 & CGTAAAAATTITGTCGACATITITGATATAT & \multirow{2}{*}{ ATG2 4779-4775 } & \multirow{2}{*}{ introduces Sall site } \\
\hline Sal11rev & ATATATCAAAAATGTCGACAAAAATTITTACG & & \\
\hline Blpl_F & GCTCAGCCAAAAGAAGAAGAC & ATG2 3016-3036 & \multirow{2}{*}{ amplification of $A T G 2$ fragment } \\
\hline Stul_R & GACAGGAGCCAGGCCTTCCAA & ATG2 4047-4027 & \\
\hline M13F & TGTAAAACGACGGCCAGT & & $\begin{array}{l}\text { universal primer complementary to } \\
\text { pGEM T-Easy, used for fusion PCR }\end{array}$ \\
\hline YK_R & CGTGTGTAGGTACAGGAATTTATTCCAGGTGG & ATG2 3552-3539 & $\begin{array}{l}\text { 5' end contains } 18 \text { nucleotide overhang } \\
\text { complementary to KIAAO404 (4716- } \\
\text { 4699), used for fusion PCR }\end{array}$ \\
\hline YK_F & TCC CAG ATC AAC AAG TIT GTA ACT TTG CTA AAG CA & ATG2 3553-3572 & $\begin{array}{l}5^{\prime} \text { end contains overhang complemen- } \\
\text { tary to KIAAO404 (4684-4698), used for } \\
\text { fusion PCR }\end{array}$ \\
\hline SalATG_R & TAACTAATTACATGACTCGAGGTC GACA & & $\begin{array}{l}\text { complementary to terminator sequence } \\
\text { of } A T G 2 \text {, introduces Sall site }\end{array}$ \\
\hline Sal3_F & CCTGGAATAAAGTCGACACTTTG & ATG2 3542-3564 & \multirow{2}{*}{ introduces Sall site } \\
\hline Sal3_R & GCAAAGTGTCGACTTIATTCC & ATG2 3565-3545 & \\
\hline 0404LBam & ACA TGG ATC CCT CGG AGA CCGCCG GGC CTG & KIAA0404 4-1 & \multirow{2}{*}{ introduces BamHI site } \\
\hline 0404UBam & CGA GGG ATC CAT GTC ACG ATG GCT GTG GCC A & KIAA0404 1-21 & \\
\hline KY_L & CCACCTGGAATAAATTCCTGTACCTACACACG & KIAA0404 4699-4716 & $\begin{array}{l}5^{\prime} \text { end contains } 14 \mathrm{nt} \text { overhang com- } \\
\text { plementary to ATG2 (3539-3552) }\end{array}$ \\
\hline SalKIA_R & ACTCGAGGTCGACTCAGTCTTGGGCAC & KIAA0404 5899-5885 & introduces Sall site to KIAA0404 \\
\hline KY_R & TTAGCAAAGTTACAAACTTGTTGA TCTGGGAGGA & KIAA0404 4698-4681 & $\begin{array}{l}5^{\prime} \text { end contains overhang to ATG2 } \\
(3568-3553)\end{array}$ \\
\hline Sacll_F & CCTCATCACCGCGGAGACC & KIAA0404 3477-3495 & complementary to KIAA0404 \\
\hline Atg94 & GAAATTGAGATGGTGCAGGATCCACAGTTGAAGTG & & introduces BamHI site after ATG9 orf \\
\hline Atg93 & GGAATTCCCGATCCCCATGGAGAGAGATGAATAC & ATG9 1-18 & $\begin{array}{l}\text { introduces Ncol site in start codon of } \\
\text { ATG9 orf }\end{array}$ \\
\hline
\end{tabular}

$\mathrm{kb}$ EcoRI-EcoRV fragment was transferred from the KS-KIAA0404-EcoRI plasmid (BS-KIAA0404 derivative), in which an EcoRI restriction site had been introduced by PCR before start codon, to pGBT9 EcoRI and SmaI restriction sites. Similarly, to construct pGBT9-KIAA0404-N the Eco47III fragment of plasmid KS-KIAA0404-EcoRI was deleted and than EcoRIEcoRV fragment was transferred to pGBT9. To obtain pACT2-ATG9, the ATG9 orf was amplified by PCR by using primers containing $\mathrm{NcoI}$ and $\mathrm{BamHI}$ restriction sites, and pGBDUAtg9 as a template, and was cloned to pACT2 NcoI and BamHI restriction sites. pACT2ATG9 sequence was confirmed.

Total protein extracts and Western blot analysis. Protein extracts were prepared as described (Baggett et al., 2003). Samples were analyzed by Western blotting using anti-GFP (Roche), anti-aminopeptidase I (anti-Ape1, kindly provided by D. Klionsky, University of Michigan, Ann Arbor, USA) and secondary anti-mouse or anti-rabbit HRP-conjugated antibodies (DACO), followed by enhanced chemiluminescence (Millipore). A representative result of two experiments is shown.

Fluorescence microscopy. For GFP fluorescence yeast were grown to the logarithmic phase in SD-leu-his medium at $30^{\circ} \mathrm{C}$. After that cells were shifted to YB-N starvation medium for 4 hours. At least 100 cells of each strain were viewed with an Eclipse (Nikon) fluorescence microscope equipped with an ORCA (Nikon) camera. Images were collected using Lucia General 5.1 software (Laboratory Imaging Ltd.).

Monitoring of authophagy by Pho8 $\Delta 60$ alkaline phosphatase test. For measurements of autophagic activity, the alkaline phosphatase test was performed as described (Noda et al., 1995). Five independent experiments were performed and means \pm S.D. (standard deviation) are shown.

Bioinformatic analyses. The non-redundant database at NCBI was scanned for sequences similar to the Atg2 protein using the PSI-Blast program (Altschul et al., 1997). Fifty fungal Atg2 orthologues were aligned using the MAFFT program (Katoh et al., 2002) and manually inspected for differences. Among human proteins, Atg2 homologues were found using the PSIBlast program (Altschul et al., 1997) with the first hits detected in the first iteration with e-values of $5 \mathrm{e}-11$ and 4e-15 for $\mathrm{hAtg} 2 \mathrm{~A}$ and $\mathrm{Atg} 2 \mathrm{~B}$, respectively. Multiple sequence alignment of yeast Atg2 with the Aspergillus nidulans AnAtg2 and human hAtg2A homologues was 


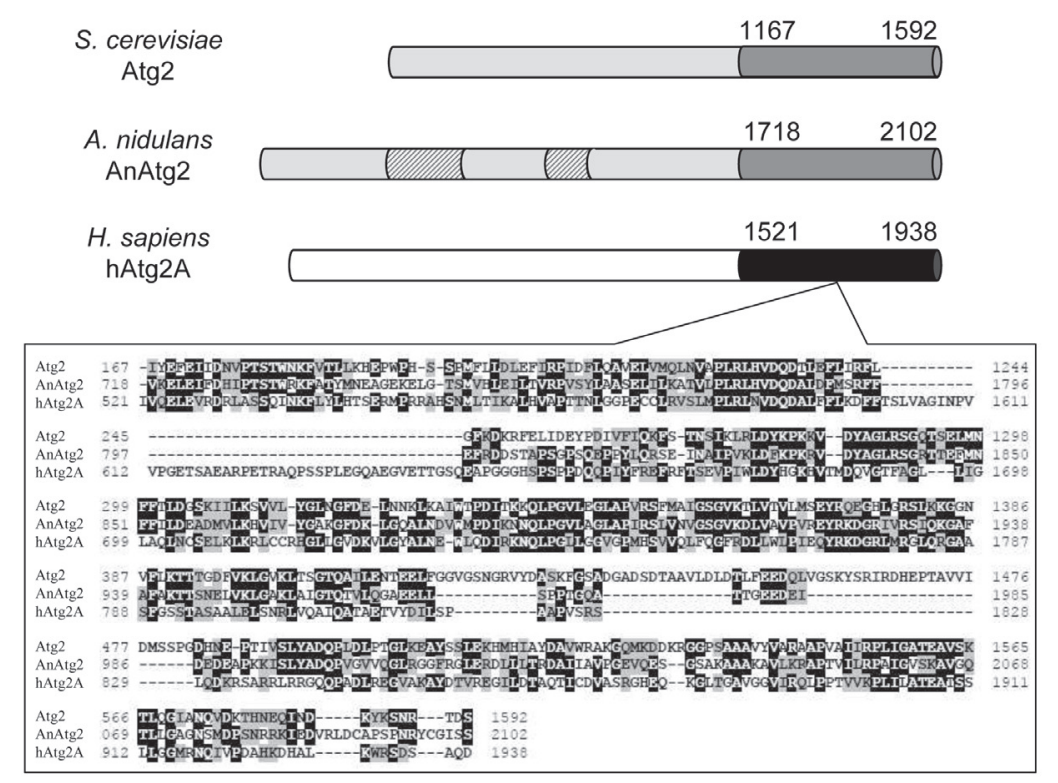

Figure 1. Atg2 domain structure and homology to other Atg2 proteins Schematic representation of domain structure of Atg2, AnAtg2 and hAtg2A. N-terminal and C-terminal fragments of fungal AnAtg2 and Atg2 are in light grey and dark grey, respectively. N-terminal fragment of hAtg2A is in white and C-terminal domain of hAtg2A in black. Regions specific to proteins of filamentous fungi are shown striped.

done using MAFFT with 1000 iterations and then formatted in BioEdit (Hall, 1999).

\section{RESULTS}

\section{Homologues of yeast Atg2 protein}

Autophagy is a process conserved in evolution from yeast to humans, and several yeast Atg proteins involved in autophagy have orthologues in human cells (Reggiori \& Klionsky, 2002). Specifically, the yeast Atg2 protein was reported to be related to the protein encoded by the human KIAA0404 gene (Shintani et al., 2001), hAtg2A. We performed in silico analysis using PSI-Blast to find homologues of yeast Atg2 in other organisms and to predict the Atg2 domain structure.

Our analysis revealed that $S$. cerevisiae Atg2 is highly homologous to Atg2 proteins from other fungi forming a fifty-strong Atg2 family with 24 members belonging to the Pezizomycotina subphylum, 22 to Saccharomycotina and 4 - to Basidiomycotina. All three subphyla seem to have quite similar Atg2s, except for large insertions in the filamentous fungi. The largest insertions are located in regions $260-480$ and $720-850$ of the filamentous fungi polypeptide sequences. The provenience and function of these regions have not been studied yet. The C-terminal region of Atg2 (aa 1167-1592) is also homologous to the C-terminal region of human hAtg2A protein (aa 1521-1938) (25\% identity, e-value 3e-12, score 72.4. using BLAST) and the corresponding region of the hAtg2B protein (aa 1644-2078) encoded by the C14orf103 gene (Fig. 1 and data not shown).

\section{KIAA0404 gene can be expressed in yeast}

The data about the cellular function of the hAtg2A protein encoded by the KIAA0404 gene was lacking, thus we used yeast to express the KIAA0404 gene and to study its potential function in autophagy. Plasmid p415-GFPKIAA0404 encoding hAtg2A protein N-terminally fused to GFP was constructed. The fusion gene was expressed from a strong constitutive GPD1 promoter. A plasmid encoding yeast Atg2 fused to GFP was also constructed to serve as a control. atg $2 \Delta$ yeast cells were transformed with p415-GFP-KIAA0404 and control plasmids (p415-GFP, p415-GFP-ATG2), grown in medium containing glucose, shifted or not to nitrogen starvation medium for 4 hours, and protein extracts were prepared and analyzed by Western blotting using anti-GFP antibody. As expected, when GFPATG2 was expressed an abundant protein product of about $220 \mathrm{kDa}$ was observed. When the GFP-KI$A$ A0404 gene was expressed the GFP-hAtg2A fusion protein of about $315 \mathrm{kDa}$ was present but its level was low and two smaller proteins of $130 \mathrm{kDa}$ and about $30 \mathrm{kDa}$ were observed, probably representing partial degradation products, the smaller one resembling GFP (Fig. 2a and data not shown). The atg2 $\triangle$ transformants expressing GFP-ATG2 or GFP-KI$A A 0404$ shifted to nitrogen starvation medium showed protein products of similar size as in growth conditions, but less abundant (not shown).

(a)

(b)
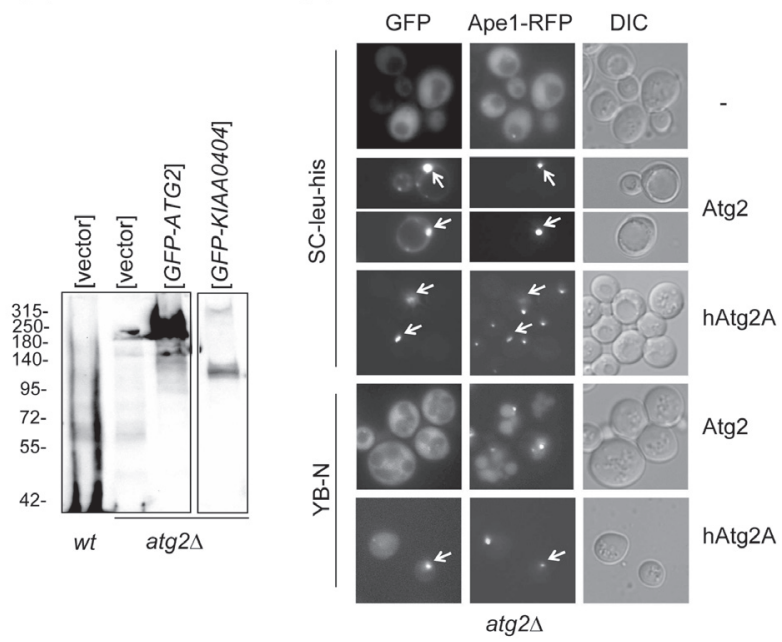

Figure 2. Expression of KIAA0404 in yeast

(a) Western blot analysis. atg2 $\triangle$ strain (YPJ1) was transformed with plasmids expressing GFP, GFP-ATG2 or GFP-KIAA0404 genes and grown in SD-leu medium to $\mathrm{OD}_{600}$ of $0.4-0.6$. Extracts were prepared and Western blot analysis was performed using anti-GFP antibody. (b) Localization of human hAtg2A in yeast cells. atg2 $\triangle$ strain (YPJ1) was transformed with plasmids expressing GFP, GFPATG2 or GFP-KIAA0404 and with plasmid expressing APE1-RFP. Transformants were grown in SC-leu-his medium to $\mathrm{OD}_{600}$ of 0.40.6 and were shifted to nitrogen starvation medium for 4 hours. Cells were viewed by epifluorescence or by DIC optics. Arrows indicate punctate structures where GFP-hAtg2A and Ape1-RFP colocalize. 


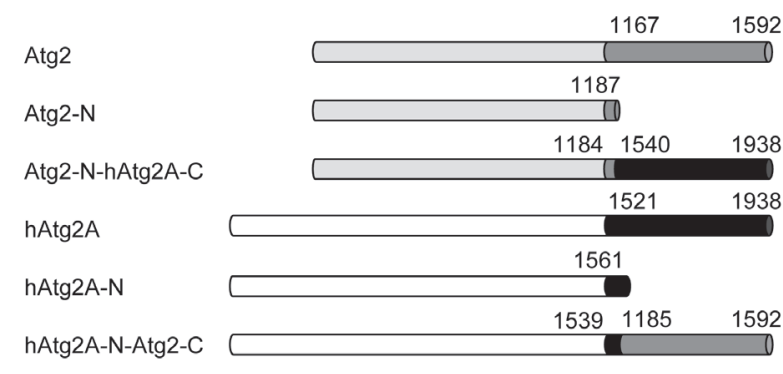

Figure 3. Schematic representation of Atg2 and hAtg2A domain structure and their constructs expressed with $\mathrm{N}$-terminally fused GFP

Fragments and domains are as in Fig. 1.

We also transformed wild type cells with the above plasmids and tested for growth on YPD, and SD-leu at three temperatures: $28^{\circ} \mathrm{C}, 34^{\circ} \mathrm{C}$ and $37^{\circ} \mathrm{C}$. Cells expressing GFP-ATG2, GFP-KIAA0404 grew similarly to control cells in these conditions (not shown). Thus, the GFP-KIAA0404 gene can be expressed in yeast cells and $\mathrm{hAtg} 2 \mathrm{~A}$ is not toxic to yeast cells in growth conditions.

\section{hAtg2A is localized in a few cytoplasmic dots and one of these dots colocalizes with Ape1-RFP, so it is recruited to the PAS}

Atg2 localizes in yeast cells to one or two punctate cytoplasmic structures in growth conditions and to one such structure adjacent to a vacuole called PAS in starvation condition (Shintani et al., 2001; Suzuki et al., 2007). PAS is the site of recruitment of membranes and cargo proteins, such as Ape1, to the growing autophagosome. So, Ape1 fused to a fluorescent tag such as red fluorescent protein (Ape1-RFP) can be used as a marker for PAS in growing cells (Kawamata et al., 2005; Suzuki et al., 2007). Ape1-RFP is localized to the vacuole in starved cells. Similarly as published, in our experiments in atg $2 \Delta$ cells in growth conditions we observed GFPAtg2 in one to three cytoplasmic dots, one of which colocalized with Ape1-RFP, the PAS marker. Also slight

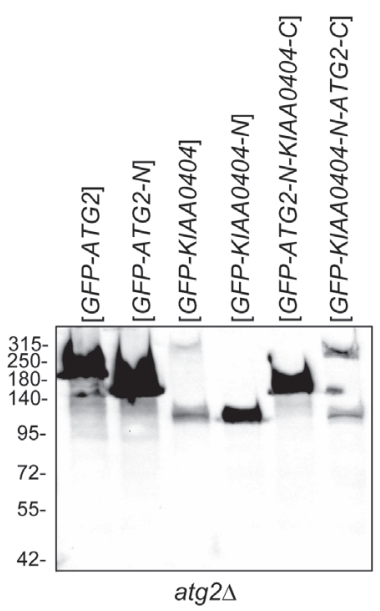

Figure 4. Cellular levels of GFP-Atg2-N, GFP-hAtg2A-N, and fusions of Atg2 with hAtg2A.

atg2 $\triangle$ strain was transformed with plasmids expressing GFP-ATG2-N, GFP-KIAA0404-N, GFP-ATG2-N-KIAA0404-C or GFP-KIAA0404-N-ATG2$C$ and grown in SD-leu medium to $\mathrm{OD}_{600}$ about 1 . Extracts were prepared and Western blot analysis was performed using anti-GFP antibody. Extracts from cells expressing GFP-ATG2 or GFP-KIAA0404 were used as a control. diffused cytoplasmic staining was observed probably due to overproduction of the protein (Fig. 2b). In starvation conditions GFP-Atg2 was cytoplasmic in most cells and some cells showed also a single dot while Ape1-RFP was vacuolar and in some cells also localized to the PAS (Fig. 2b).

Cellular localization of the human GFP-hAtg2A protein was observed in atg $2 \Delta$ cells, and was compared with the localization of Ape1-RFP, GFP-Atg2 and of GFP alone. In growth conditions GFP was diffused in the cytoplasm while the GFP-hAtg2A protein was localized to 1-3 punctate structures (Fig. 2b). One of them colocalized with the Ape1-RFP punctum in about $60 \%$ of atg $2 \Delta$ cells in which both GFP and RFP signals were visible, suggesting that GFP-hAtg2A could be recruited to the PAS. In control experiments employing atg1 $\Delta$ cells in which PAS does not form we did not find colocalization of GFP-Atg2A and Ape1-RFP, which supports this conclusion (not shown). The identity of the punctate structures other than PAS containing GFP-Atg2A is unknown. In starvation conditions GFP-hAtg $2 \mathrm{~A}$ was cytoplasmic in $95 \%$ of cells and $5 \%$ of cells showed it in one punctate structure (Fig. 2b). The cytoplasmic staining may also derive from the partial degradation product, GFP. In these cells Ape1-RFP was found in the PAS, not in the vacuole, indicating a lack of complementation of the autophagy defects of atg $2 \triangle$ by GFP-KIAA0404.

\section{$\mathrm{N}$-terminal fragments of Atg2 and hAtg2A are sufficient for PAS recruitment but do not support Cvt transport of Ape1-RFP to the vacuole}

Since $\mathrm{hAtg} 2 \mathrm{~A}$ protein, despite being partially recruited to the PAS, did not complement the Cvt defect of $\operatorname{atg} 2 \Delta$ cells, in which it differed from the yeast Atg2, several questions arose. Which part of Atg2 is required for PAS recruitment and for functioning in the Cvt pathway? Is the function of the C-terminus of Atg2 conserved? Can the C-terminal part of Atg2 be replaced with a homologous fragment of hAtg $2 \mathrm{~A}$ ? To answer these questions several plasmids were constructed and the cellular level and localization of protein products encoded was analyzed. These were plasmids expressing: GFP-ATG2-N (aa 1-1184) devoid of a fragment encoding the C-terminus of Atg2, GFP-KIAA0404-N (aa 1-1561) devoid of a fragment encoding the C-terminus of hAtg2A, GFP-ATG2-NKIAA0404-C fusion in which the fragment encoding the C-terminus of Atg2 (aa 1185-1592) was replaced by a fragment encoding the C-terminus of hAtg2A (aa 1540-1938), and GFP-KIAA0404-N-ATG2-C encoding a fusion protein of the $\mathrm{N}$-terminal part of hAtg $2 \mathrm{~A}$ (aa 1-1539) fused to the C-terminal part of Atg2 (aa 1185-1592) (Fig. 3). The site of fusion of the Atg2 and $\mathrm{hAtg} 2 \mathrm{~A}$ proteins was chosen between amino acids KR which are identical in both proteins and are located in the first most conserved block of amino acids (see Fig. 1). Expression of these fusion genes in atg2 $\Delta$ strain was analyzed by Western blotting and compared with the expression level of GFP-ATG2 and GFP-KIAA0404 in growth and starvation conditions (Fig. 4 and data not shown). The expression of GFP-ATG2-N was very efficient and comparable to that of GFP-ATG2, and Atg2-N protein of expected size of about $170 \mathrm{kDa}$ was observed. The expression of GFP-KIAA0404-N was less efficient and hAtg2A-N migrated faster than expected, of about $130 \mathrm{kDa}$ instead of $220 \mathrm{kDa}$. This size was similar to the major 


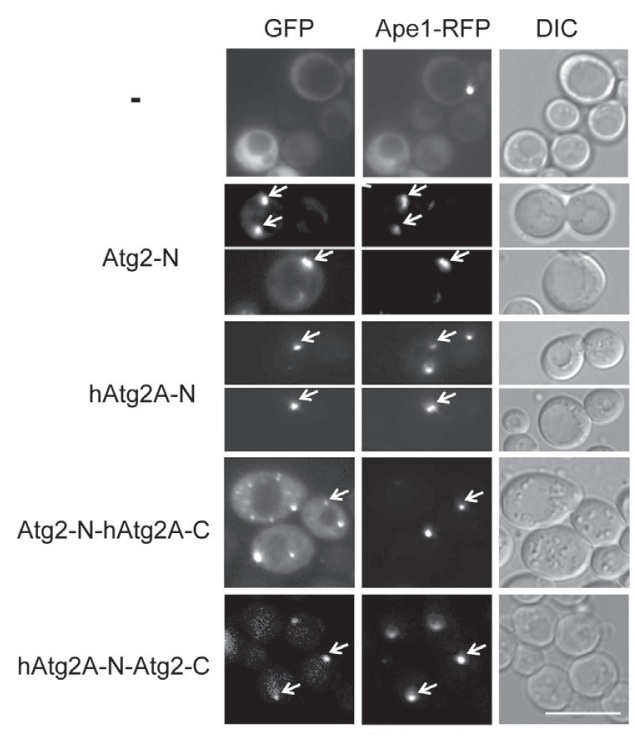

SC-leu-his
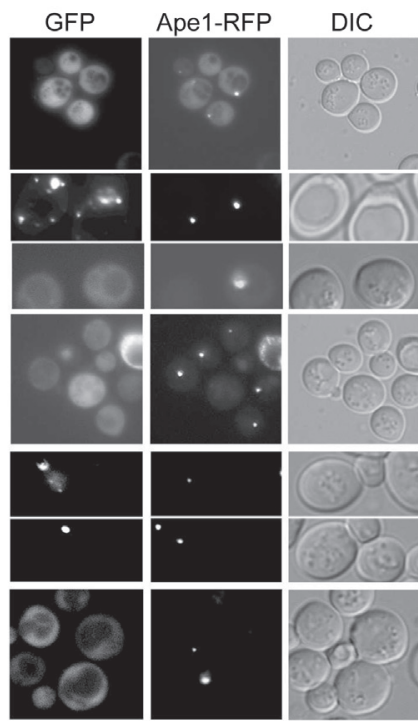

YB-N

Figure 5. Localization of GFP-Atg2-N and GFP-hAtg2A-N fragments, and GFP-Atg2-NhAtg2A-C and GFP-hAtg2A-N-Atg2-C fusions.

atg $2 \triangle$ strain was transformed with plasmids expressing GFP, GFP-ATG2-N, GFP-KIAA0404-N, GFP-ATG2-N-KIAA0404-C or GFP-KIAA0404-N-ATG2-C and with plasmid expressing APE1-RFP. Transformants were grown in SC-leu-his medium to $\mathrm{OD}_{600}$ of $0.4-0.6$ and were shifted to nitrogen starvation medium for 4 hours. Cells were viewed by epifluorescence or by DIC optics. Arrows indicate punctate structures where GFP and RFP signal colocalize. Scale bar, $10 \mu \mathrm{m}$.

(a)

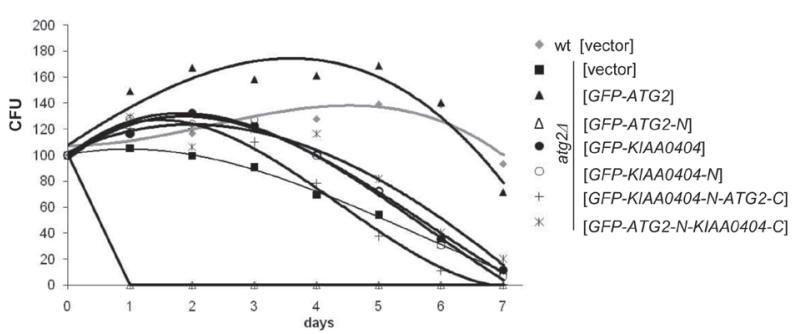

(b)

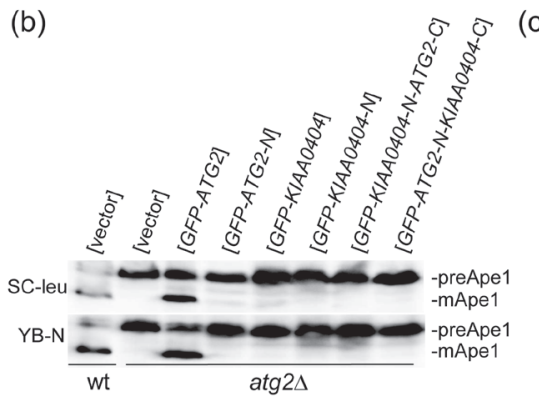

(c) 70

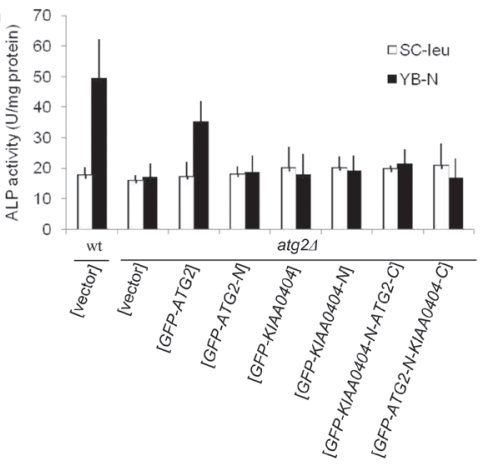

Figure 6. Lack of complementation of atg2 $\triangle$ autophagy defects by GFP-ATG2-N, GFPKIAA0404, GFP-KIAA0404-N and fusions of KIAA0404 with ATG2.

(a) Survival of $\operatorname{atg} 2 \triangle$ (YPJ1) trasformants during starvation. Wild type strain transformed with empty vector for control and atg2 $\triangle$ cells transformed with plasmids expressing GFP, GFP-ATG2, GFP-ATG2-N, GFP-KIAA0404, GFP-KIAA0404-N, GFP-ATG2-N-KIAA0404-C or GFPKIAA0404-N-ATG2-C were grown in SC-leu, shifted to YB-N, and tested for viability after 1 to 7 days of incubation. Colony forming units (CFU) were scored. (b) Complementation of atg2 2 (YPJ1) defect of Cvt pathway. Same set of transformants as in (a) was grown in SD-leu, shifted to YB-N for 4 hours, then extracts were prepared and analyzed by Western blotting using anti-Ape1 antibody. (c) Complementation of atg2 $(\mathrm{YPJ} 1)$ defect of nonselective autophagy. Wild type cells transformed with empty vector and atg2 $\triangle$ cells expressing GFP, GFP-ATG2, GFP-ATG2-N, GFP-KIAA0404, GFP-KIAA0404-N, GFP-ATG2-N-KIAA0404-C or GFP-KIAA0404-N-ATG2-C were grown in SD-leu, shifted or not to YB-N for 4 hours, then extracts were prepared and Pho8 $\Delta 60$ test was performed.

band of GFP-hAtg2A representing partial degradation product, indicating that the C-terminal part of hAtg2A could be unstable in yeast. The apparent molecular mass of the protein product of GFPATG2-N-KIAA0404-C was about $180 \mathrm{kDa}$ and was lower than expected $(220 \mathrm{kDa})$, again indicating an instability of the C-terminus of hAtg2A. The full length product of GFP-KIAA0404-N-ATG2-C showed the expected size of about $260 \mathrm{kDa}$ but a partial degradation product of about $130 \mathrm{kDa}$ was also observed. We cannot exclude the possibility that some of these proteins migrate aberrantly and in fact are not even partially degraded. After a shift to starvation conditions all these proteins were present but much less abundant (not shown). The localization of the N-terminal parts of the proteins, Atg2-N and hAtg2A-N, expressed from plasmids described above was observed in atg2. strain in growth and nitrogen starvation conditions and compared with the localization of GFP-Atg2, GFP-hAtg2A and Ape1-RFP. GFP-Atg2-N was present in 1-3 dots and slightly diffused in the cytoplasm, similarly as GFP-Atg2. One of these dots colocalized with Ape1-RFP in about $50 \%$ of cells indicating that the N-terminal fragment of Atg2 is sufficient for PAS recruitment. Interestingly, about $20 \%$ of cells showed more that one Ape1-RFP dot indicating that more than one PAS could be present in some cells or Ape2RFP could be located also in other punctate structure in this condition (Fig. 5). Observation of GFPAtg2-N in starvation conditions showed cytoplasmic localization or in 1-5 dots, and Ape1-RFP was localized in the PAS. On the other hand, GFP-hAtg2A-N was localized in one or two dots and slightly diffused in the cytoplasm and colocalized with Ape1-RFP in $60 \%$ of cells indicating that the $\mathrm{N}$-terminal part of hAtg2A is also sufficient for PAS recruitment. Ape1-RFP was localized in two dots in some of these cells.

Fusion proteins GFP-Atg2-NhAtg2A-C and GFP-hAtg2A-NAtg2-C were localized in few dots or one, respectively, and slightly diffused in the cytoplasm in atg2 4 cells in growth conditions (Fig. 5). One of these dots colocalized with Ape1-RFP, and the recruitment to PAS was more efficient for the 
hAtg2A-N-Atg2-C fusion. In starvation conditions GFPAtg2-N-hAtg2A-C was found in few cytoplasmic dots while GFP-hAtg2A-N-Atg2-C was in the dots and the cytoplasm. Ape1-RFP localized exclusively to the PAS, not to the vacuole, in cells expressing both fusion proteins showing a lack of complementation of Cvt defects of $\operatorname{atg} 2 \Delta$ (Fig. 5).

\section{Intact KIAA0404 and fusions containing fragments of KIAA0404 do not complement defects of atg2 $\Delta$ cells in survival upon starvation, preApe1 maturation and nonselective autophagy}

The involvement of $\mathrm{hAtg} 2 \mathrm{~A}$ protein in yeast authophagy could be assessed by investigating complementation of individual phenotypes of atg2 $\Delta$ cells by GFP-KIAA0404 or its derivatives. The atg $2 \Delta$ cells do not survive for longer than five days under nitrogen starvation conditions when glucose is present in the medium, they show a block in preApe1 maturation and a defect in nonselective autophagy measured by Pho8 860 test (Shintani et al., 2001; Wang et al., 2001). First we analyzed the complementation of the $\operatorname{atg} 2 \triangle$ viability defects by GFP-KIAA0404, its N-terminal fragment GFP-KIAA-0404-N, and the fusions GFPKIAA0404-N-ATG2-C and GFP-ATG2-N-KIAA0404-C relative to the complementation by GFP-ATG2 and GFP-ATG2-N. The respective transformants were grown in SC-leu, transferred to nitrogen starvation medium for seven days and tested for viability by scoring colony forming units after each day. The wild type control strain remained viable throughout the experiment while the $\operatorname{atg} 2 \Delta$ cells bearing the empty plasmid died after six days, in good agreement with published data. Plasmids expressing GFP-ATG2 fully complemented the $\operatorname{atg} 2 \Delta$ defect, cells remained viable, similarly as wild type strain, after 7 days of starvation. Survival of transformants bearing plasmids expressing GFP-KIAA0404, GFP-KIAA0404-N or the fusion genes declined during starvation and was similar to the atg $2 \Delta$ control cells after 7 days of starvation (Fig. 6a). This result indicates that expressed KIAA0404 gene, its 5' fragment or fusions do not complement the atg2 $\triangle$ defects in survival. Surprisingly, GFP-ATG2-N was very toxic to atg $2 \Delta$ cells, they did not survive even the first day of starvation. However, the KIAA0404 fragment encoding homologous C-terminal part of hAtg2A fused with GFP$A T G 2-N$ (resulting in GFP-ATG2-N-KIAA0404-C) prevented this toxicity, similarly as the respective native fragment of ATG2. Although we do not observe full length fusion protein expressed from GFP-ATG2-N$K I A A 0404-C$ in our Western blot analysis (Fig. 4), this protein may be present and function in the cells and be partially degraded during protein extraction.

Another test performed for complementation of atg2 $\triangle$ by GFP-KIAA0404 was the Western blot test for preApe1 maturation. In growth conditions in wild type cells preApe1 is transported via the Cvt pathway to the vacuole where it is proteolytically processed. This pathway is blocked in atg2 $\Delta$ strains (Shintani et al., 2001; Wang et al., 2001). The maturation of preApe1 was analyzed by Western blotting in the set of transformants described above grown in SD-leu medium and shifted to nitrogen starvation conditions. Neither GFP-KIAA0404, its fragment GFP-KIAA0404-N nor the GFP-KIAA0404$N-A T G 2-C$ or GFP-ATG2-N-KIAA0404-C fusions restored preApe1 maturation in atg $2 \Delta$, in conditions when GFP-ATG2 expressed from the plasmid was fully functional (Fig. 6b). These results indicate that the human hAtg2A protein or its fragments cannot take over the yeast Atg2 function in the Cvt pathway. This is in agreement with our Ape1-RFP localization results in the atg2 $\triangle$ strain bearing KIAA0404-containing plasmids, described above (see Fig. 5). Similarly, preApe1 maturation was not restored in atg2 $\Delta$ cells expressing GFP-ATG2-N, showing that the Atg2-N fragment is not sufficient for function. (a)

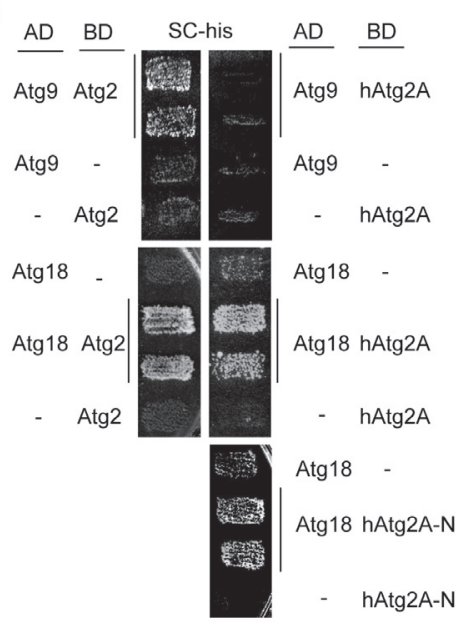

(b)

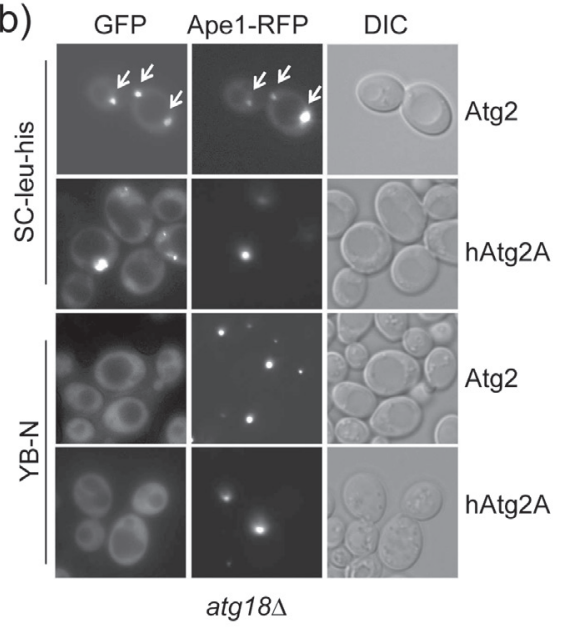

Figure 7. hAtg2A interacts with Atg18 and requires Atg18 for recruitment to the PAS.

(a) Interaction of Atg2 and hAtg2A with Atg9 and Atg18. Two-hybrid tester strain (PJ69-4A) was Co-transformed with plasmids expressing activating domain (AD)-fused Atg9 or Atg18 and Atg2, hAtg $2 A$ or hAtg2A-N fused with the DNA binding domain (BD) or with empty vectors (-). Interactions were monitored by the ability of cells to grow on plates lacking histidine and containing 5-10 mM 3-aminotriazol (SC-his). (b) Localization of Atg2 and hAtg2A in atg18D strain. atg18 strain was transformed with plasmids expressing GFP-ATG2 or GFP-KIAA0404 and with plasmid bearing APE1-RFP. Transformants were grown in SC-leu-his medium to $\mathrm{OD}_{600}$ of $0.4-0.6$ and were shifted to nitrogen starvation medium for 4 hours. Cells were viewed by epifluorescence or by DIC optics. Arrows indicate punctate structures where GFP and RFP signals colocalize.
We also tested Pho8 860 activity, a measure of nonselective autophagy, in the set of transformants described above. Wild type cells transformed with empty vector responded to nitrogen starvation by increasing the activity of Pho $8 \Delta 60$ more than 2-fold while in atg $2 \Delta$ cells this activity was similar as in growth conditions (Fig. 6c), in agreement with previously published data (Shintani et al., 2001). Expression of GFP-ATG2 complemented this phenotype, the Pho8 800 activity was significantly increased upon starvation. This complementation was lost when GFP-ATG2-N was expressed, indicating that the N-terminal fragment of Atg2 is not sufficient to function in nonselective autophagy. Complementation was also not observed in atg2 $\triangle$ cells expressing GFP-KIAA0404, its fragment GFP-KIAA0404-N or fusions with fragments of 
ATG2, showing that hAtg2A does not function in nonselective autophagy in yeast.

\section{hAtg2A interacts with Atg18 but does not interact with Atg9 in two-hybrid system}

Atg2 was shown to interact with the membrane protein Atg9 (Wang et al., 2001) and lipid binding protein Atg18 (Obara et al., 2008b; Nair et al., 2010) and to be required for Atg9 cycling between PAS and non-PAS peripheral compartment (Reggiori et al., 2004). Therefore, we tested if these interactions of Atg2 could be reproduced in yeast two-hybrid system and if hAtg2A also interacted with the same proteins. Transformation of a tester strain with plasmids bearing gene fusions of $G A L A_{A D}$ with $A T G 9$ or ATG18 and plasmids bearing $G A L A_{B D}$ fused with $A T G 2$ or KIAA0404 followed by the analysis of transformants for growth on SC-his plates showed that Atg2 interacted with both Atg9 and Atg18 in this system (Fig. 7a). hAtg2A did not interact with Atg9 but an interaction with Atg18 was observed (Fig. 7a). The interaction of hAtg2A with Atg18 suggests that Atg18 is probably one of the proteins mediating recruitment of hAtg2A to PAS. Consistent with the fluorescence microscopy studies showing hAtg2A-N recruitment to the PAS, the N-terminal fragment of hAtg2A was also able to interact with Atg18 in two-hybrid system (Fig. 7a).

To analyze the involvement of Atg18 in hAtg2A recruitment to PAS we observed the cellular localization of GFP-hAtg2A in the atg18 4 strain in growth and nitrogen starvation conditions and compared it with localization of Atg2. We found that in growth conditions Atg2 was present in one or two cytoplasmic dots and colocalized with Ape1-RFP1 in most cells. In some cells Ape1RFP was found in two dots, as before. In contrast, hAtg2A was found in one to five dots, but none of those dots colocalized with Ape1-RFP. Upon nitrogen starvation Atg2 and hAtg2A diffused in the cytoplasm while Ape1-RFP was in PAS and not in the vacuole (Fig. 7b). These results indicate that ATG18 is important for PAS localization of hAtg2A in both growth and starvation conditions. It was previously reported that ATG18 is required for PAS recruitment of Atg2 in autophagy-inducing conditions (rapamycin treatment) (Suzuki et al., 2007) and our results are consistent with those findings. We also indicated that some factors may help to recruit Atg2 to Ape1-RFP-containing dots in growing atg18 $\Delta$ cells.

\section{DISCUSSION}

The function of human hAtg2A protein encoded by KIAA0404 has not been reported to date. When this work was in progress several proteins interacting with hAtg2A were identified, including some proteins involved in autophagy in humans (Behrends et al., 2010). Here we studied the homology of Atg2 and hAtg2A and used yeast as a model organism to test the possible involvement of $\mathrm{hAtg} 2 \mathrm{~A}$ in autophagy. We showed that GFP-KIAA0404 could be expressed in yeast and its protein product, GFP-hAtg2A, was recruited to the PAS. This recruitment is probably mediated by Atg18 which interacted with hAtg2A in the two hybrid system. Human hAtg2A did not support growth of atg $2 \Delta$ cells in starvation conditions and could not substitute for the yeast Atg2 protein in the Cvt pathway or non-selective autophagy. Moreover, we showed that the N-terminal fragments of Atg2 or of hAtg2A were sufficient for the recruitment to the PAS but they did not function in autophagy. Also, the C-terminal fragment of Atg2 could not be functionally substituted for homologous C-terminal fragment of hAtg2A and vice versa. Surprisingly, we also found that Atg2-N was toxic to yeast cells in starvation conditions and this toxicity was prevented by fusion with the hAtg2A C-terminus.

Human hAtg2A and yeast Atg2 show only limited homology, restricted to the C-terminal domain. However, the hAtg2A protein did localize to cytoplasmic punctate structures, some of which colocalized with Ape1-RFPmarked PAS, similarly as did yeast Atg2. One of the proteins mediating PAS recruitment of hAtg $2 \mathrm{~A}$ could be Atg18 since these proteins interacted in the two-hybrid assay in our experiments and deletion of ATG18 abolished PAS localization of hAtg2A. Atg18 binds Atg2 independently of its phosphatidylinositol-3-phosphate (PI3P)-binding ability (Obara et al., 2008b), and this complex is recruited to PAS by PI3P, in which PAS is enriched (Obara et al., 2008a) and by other factors (Suzuki et al., 2007). One such factor is Atg9 (Wang et al., 2001). However, in contrast to Atg2, hAtg2A did not bind Atg9 in two-hybrid system suggesting that other proteins must also contribute to the hAtg $2 \mathrm{~A}$ recruitment to PAS. Very recently, studies of proteomic network organization of the human autophagy system have identified nine proteins interacting with hAtg2A (Behrends et al., 2010). Among them are hAtg2B, and WIPI1 and WDR45, two WD40 domain-containing proteins homologous to Atg18. This supports our finding and indicates that the Atg2-Atg18 interaction is conserved in evolution from yeast to humans. Moreover, hAtg2A was found among interacting proteins when any of the Atg8 human homologues (GABARAP, GABARAPL1, GABARAPL2, MAP1LC3B, MAP1LC3B) was used as a bait in these proteomic studies (Behrends et al., 2010). This suggests that Atg 8 may contribute to the PAS recruitment of hAtg2A in yeast. However, PAS assembly studies showed that Atg8 is not required for Atg2 PAS localization (Suzuki et al., 2007) and rather the Atg18-Atg2 complex facilitates PAS recruitment of lipidated Atg8 (Nair et al., 2010), not the opposite. We therefore hypothesize that Atg18 could be the only protein required for the PAS localization of hAtg2A.

The N-terminal part of hAtg2A, also similarly as the $\mathrm{N}$-terminal part of Atg2, was sufficient for the punctate structure-localization and PAS recruitment, indicating that this sequence, although not showing homology, has retained its PAS-addressing function. However, this PAS localization was not sufficient to support hAtg2A, hAtg2A-N or Atg2-N function in selective and nonselective autophagy. Therefore, the C-terminal domain of Atg 2 must be important for the function in autophagy but not necessary for Atg2 localization. The C-terminal domain of $\mathrm{hAtg} 2 \mathrm{~A}$ seems rather unstable in yeast cells since it was removed in some pool of hAtg $2 \mathrm{~A}$ and a shorter form, similar in size to hAtg $2 \mathrm{~A}-\mathrm{N}$, predominated on Western blots. This could be one of the reasons of the lack of complementation of atg $2 \Delta$ defects by GFP-KIAA0404. On the other hand, the amount of full length $\mathrm{hAtg} 2 \mathrm{~A}$ could be nominally sufficient to function but the C-terminal domain of hAtg2A could lack some important protein interactions. However, the presence of $\mathrm{hAtg} 2 \mathrm{~A}-\mathrm{C}$ in fusion with Atg2-N prevented its toxicity and kept Atg2-N-hAtg2A-C in the punctate structures in starvation conditions. We tested if replacing the C-terminal domain of hAtg2A affected the function. Although the fusion protein $\mathrm{hAtg} 2 \mathrm{~A}-\mathrm{N}-\mathrm{Atg} 2-\mathrm{C}$ was more 
stable than hAtg2A and was recruited to the PAS, it still was not functional. So, the C-terminal Atg2 domain can properly function only when attached to the N-terminus of Atg2. Similarly, the C-terminal domain of hAtg2A can not functionally substitute for the C-terminal domain of Atg2 even though these domains can regulate each other's function. Some human proteins, GAS41 for example, are unable to complement phenotypes of the loss of a yeast homologue but can provide a conserved domain which is able to functionally substitute for the yeast domain (Wang et al., 2009). In our case only the combination of the $\operatorname{Atg} 2-\mathrm{N}$ and $A \operatorname{tg} 2-\mathrm{C}$ domains provides the essential functions necessary for autophagy and cell survival upon starvation.

Our results on the survival of atg2 $\triangle$ expressing GFPATG2-N pointed to previously unreported effects of this overproduced Atg2 fragment on cells upon starvation. These cells did not survive even one day of starvation, they also grew slower in growth conditions in liquid medium compared to empty plasmid-bearing cells (not shown). The mechanism of this toxicity is unknown at present but is an interesting subject for further investigation.

In our localization studies we used Ape1-RFP as a marker for PAS. However, Ape1-RFP is found in two cytoplasmic dot structures in some cells in our and other studies (Suzuki et al., 2007). Considering that there is only one PAS in the yeast cell (Xie \& Klionsky, 2007) the second Ape1-RFP complex must be located in a non-PAS compartment. Since PAS is characterized by the presence of Atg proteins we assumed that sites where Atg2 or hAtg2A colocalized with Ape1-RFP is the PAS. mCherry-Atg8, another PAS marker, also localized in more that one dot in our experimental conditions, in cells expressing GFP-ATG2 from GPD1 promoter (not shown), rising the possibility that overproduction of GFP-Atg2, or ectopic production of GFP-hAtg2A, may somehow affect PAS formation or Ape1-RFP/mCherryAtg8 recruitment to PAS.

Recycling between PAS and an Atg9-containing compartment adjacent to mitochondria has been observed for transmembrane domain-containing Atg9 protein which interacts with Atg2, and Atg2 is required for this recycling (Reggiori et al., 2005; Mari \& Reggiori, 2007; Mari et al., 2010). We observed the Atg2-Atg9 interaction by using the two-hybrid system. However, we were unable to map the interaction site in Atg2 since both Cand $\mathrm{N}$-terminal truncations abolished the binding (not shown). Considering our finding that hAtg2A did not interact with Atg9 in yeast two-hybrid system it is possible that $\mathrm{hAtg} 2 \mathrm{~A}$, even recruited to PAS, cannot perform the Atg9-recycling function. This could be a possible reason for the lack of complementation of atg $2 \Delta$ by KIA $A 0404$.

To conclude, our results showed that human hAtg2A is recruited to the PAS in yeast, possibly by interaction of its N-terminal fragment with Atg18 protein. Thus, even though hAtg2A did not function in autophagy in yeast, it is probably involved in autophagy in mammalian cells, but further work will be required to find out if indeed hAtg2A has such a role in human cells.

\section{Acknowledgements}

We thank Drs Y. Ohsumi (Tokyo Institute of Technology, Yokohama, Japan) and M. Thumm (University of Gottingen, Germany) for strains and plasmids, and D.J. Klionsky (University of Michigan, Ann Arbor, USA) for plasmids and anti-Ape1 antibody, P. Jedrzejak for strains and J. Kaminska for critical reading of the manuscript.

This work was supported by the European Union grant Ubiregulators, Marie Curie Research Training Network MRTN-CT-2006-034555 to T.Ż. D.R. is a fellow of the Ubiregulators.

\section{REFERENCES}

Altschul SF, Madden TL, Schaffer AA, Zhang J, Zhang Z, Miller W, Lipman DJ (1997) Gapped BLAST and PSI-BLAST: a new generation of protein database search programs. Nucleic Acids Res 25: 3389-3402.

Baggett JJ, D'Aquino KE, Wendland B (2003) The Sla2p talin domain plays a role in endocytosis in Saccharomyces cerevisiae. Genetics 165: 1661-1674.

Behrends C, Sowa ME, Gygi SP, Harper JW (2010) Network organization of the human autophagy system. Nature 466: 68-76.

Bergamini E, Cavallini G, Donati A, Gori Z (2004) The role of macroautophagy in the ageing process, anti-ageing intervention and ageassociated diseases. Int J Biochem Cell Biol 36: 2392-2404.

Foury F (1997) Human genetic diseases: a cross-talk between man and yeast. Gene 195: 1-10.

Goldberg AL (2003) Protein degradation and protection against misfolded or damaged proteins. Nature 426: 895-899.

Gozuacik D, Kimchi A (2004) Autophagy as a cell death and tumor suppressor mechanism. Oncogene 23: 2891-2906.

Hall TA (1999) BioEdit: a user firendly biological sequence alignment editor and analysis program from Windows 95/98/NT. Nucleis Acids Symposium Series 41: 95-98.

Hershko A, Ciechanover A (1998) The ubiquitin system. Annu Rev Biochem 67: 425-479.

James P, Halladay J, Craig EA (1996) Genomic libraries and a host strain designed for highly efficient two-hybrid selection in yeast. Genetics 144: 1425-1436.

Kabeya Y, Kawamata T, Suzuki K, Ohsumi Y (2007) Cis1/Atg31 is required for autophagosome formation in Saccharomyces cerevisiae. Biochem Biophys Res Commun 356: 405-410.

Katoh K, Misawa K, Kuma K, Miyata T (2002) MAFFT: a novel method for rapid multiple sequence alignment based on fast Fourier transform. Nucleic Acids Res 30: 3059-3066.

Kawamata T, Kamada Y, Suzuki K, Kuboshima N, Akimatsu H, Ota S, Ohsumi M, Ohsumi Y (2005) Characterization of a novel autophagy-specific gene, ATG29. Biochem Biophys Res Commun 338: 1884-1889.

Klionsky DJ, Cueva R, Yaver DS (1992) Aminopeptidase I of Saccharomyces cerevisiae is localized to the vacuole independent of the secretory pathway. J Cell Biol 119: 287-299.

Klionsky DJ, Ohsumi Y (1999) Vacuolar import of proteins and organelles from the cytoplasm. Annu Rev Cell Dev Biol 15: 1-32.

Klionsky DJ, Cregg JM, Dunn WA Jr, Emr SD, Sakai Y, Sandoval IV, Sibirny A, Subramani S, Thumm M, Veenhuis M, Ohsumi Y (2003) A unified nomenclature for yeast autophagy-related genes. Dev Cell 5: 539-545.

Kusama Y, Sato K, Kimura N, Mitamura J, Ohdaira H, Yoshida K (2009) Comprehensive analysis of expression pattern and promoter regulation of human autophagy-related genes. Apoptosis 14: 11651175.

Lynch-Day MA, Klionsky DJ (2010) The Cvt pathway as a model for selective autophagy. FEBS Lett 584: 1359-1366.

Mari M, Reggiori F (2007) Atg9 trafficking in the yeast Saccharomyces cerevisiae. Autophagy 3: 145-148.

Mari M, Griffith J, Rieter E, Krishnappa L, Klionsky DJ, Reggiori F (2010) An Atg9-containing compartment that functions in the early steps of autophagosome biogenesis. J Cell Biol 190: 1005-1022.

Mumberg D, Muller R, Funk M (1995) Yeast vectors for the controlled expression of heterologous proteins in different genetic backgrounds. Gene 156: 119-122.

Nair U, Cao Y, Xie Z, Klionsky DJ (2010) Roles of the lipid-binding motifs of Atg18 and Atg21 in the cytoplasm to vacuole targeting pathway and autophagy. J Biol Chem 285: 11476-11488.

Niedenthal RK, Riles L, Johnston M, Hegemann JH (1996) Green fluorescent protein as a marker for gene expression and subcellular localization in budding yeast. Yeast 12: 773-786.

Noda T, Matsuura A, Wada Y, Ohsumi Y (1995) Novel system for monitoring autophagy in the yeast Saccharomyces cerevisiae. Biochem Biophys Res Commun 210: 126-132.

Obara K, Noda T, Niimi K, Ohsumi Y (2008a) Transport of phosphatidylinositol 3-phosphate into the vacuole via autophagic membranes in Saccharomyces cerevisiae. Genes Cells 13: 537-547.

Obara K, Sekito T, Niimi K, Ohsumi Y (2008b) The Atg18-Atg2 complex is recruited to autophagic membranes via phosphatidylinosi- 
tol 3-phosphate and exerts an essential function. I Biol Chem 283: 23972-23980.

Petranovic D, Nielsen J (2008) Can yeast systems biology contribute to the understanding of human disease? Trends Biotechnol 26: $584-590$.

Reggiori F, Klionsky DJ (2002) Autophagy in the eukaryotic cell. Eukaryot Cell 1: 11-21.

Reggiori F, Tucker KA, Stromhaug PE, Klionsky DJ (2004) The Atg1Atg13 complex regulates Atg9 and Atg23 retrieval transport from the pre-autophagosomal structure. Dev Cell 6: 79-90.

Reggiori F, Shintani T, Nair U, Klionsky DJ (2005) Atg9 cycles between mitochondria and the pre-autophagosomal structure in yeasts. Autophagy 1: 101-109.

Sherman F (2002) Getting started with yeast. Methods Enyymol 350: 3-41.

Shintani T, Suzuki K, Kamada Y, Noda T, Ohsumi Y (2001) Apg2p functions in autophagosome formation on the perivacuolar structure. J Biol Chem 276: 30452-30460.

Suzuki K, Ohsumi Y (2010) Current knowledge of the pre-autophagosomal structure (PAS). FEBS Lett 584: 1280-1286.

Suzuki K, Kirisako T, Kamada Y, Mizushima N, Noda T, Ohsumi Y (2001) The pre-autophagosomal structure organized by concerted functions of APG genes is essential for autophagosome formation. EMBO J 20: 5971-5981.

Suzuki K, Kubota Y, Sekito T, Ohsumi Y (2007) Hierarchy of Atg proteins in pre-autophagosomal structure organization. Genes Cells 12: 209-218.

van der Vaart A, Mari M, Reggiori F (2008) A picky eater: exploring the mechanisms of selective autophagy in human pathologies. Traffic 9: 281-289.

Voth WP, Jiang YW, Stillman DJ (2003) New 'marker swap' plasmids for converting selectable markers on budding yeast gene disruptions and plasmids. Yeast 20: 985-993.

Wang AY, Schulze JM, Skordalakes E, Gin JW, Berger JM, Rine J, Kobor MS (2009) Asf1-like structure of the conserved Yaf9 YEATS domain and role in H2A.Z deposition and acetylation. Proc Natl Acad Sci USA 106: 21573-21578.

Wang CW, Kim J, Huang WP, Abeliovich H, Stromhaug PE, Dunn WA Jr, Klionsky DJ (2001) Apg2 is a novel protein required for the cytoplasm to vacuole targeting, autophagy, and pexophagy pathways. J Biol Chem 276: 30442-30451.

Xie Z, Klionsky DJ (2007) Autophagosome formation: core machinery and adaptations. Nat Cell Biol 9: 1102-1109. 